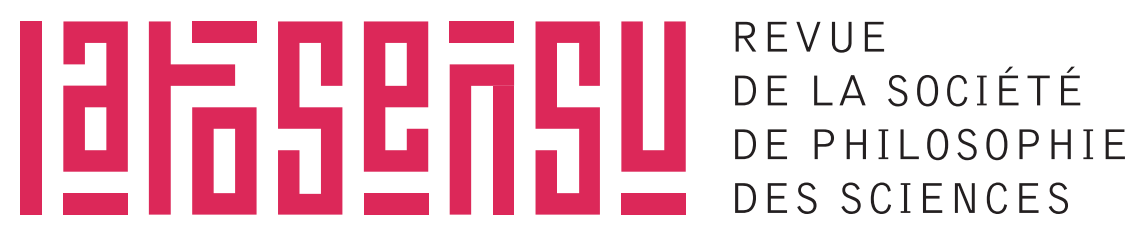

Vol $5 N^{\circ} 12018$

DOI http://dx.doi.org/10.20416/lsrsps.v5i1.4

Ignace Yapi
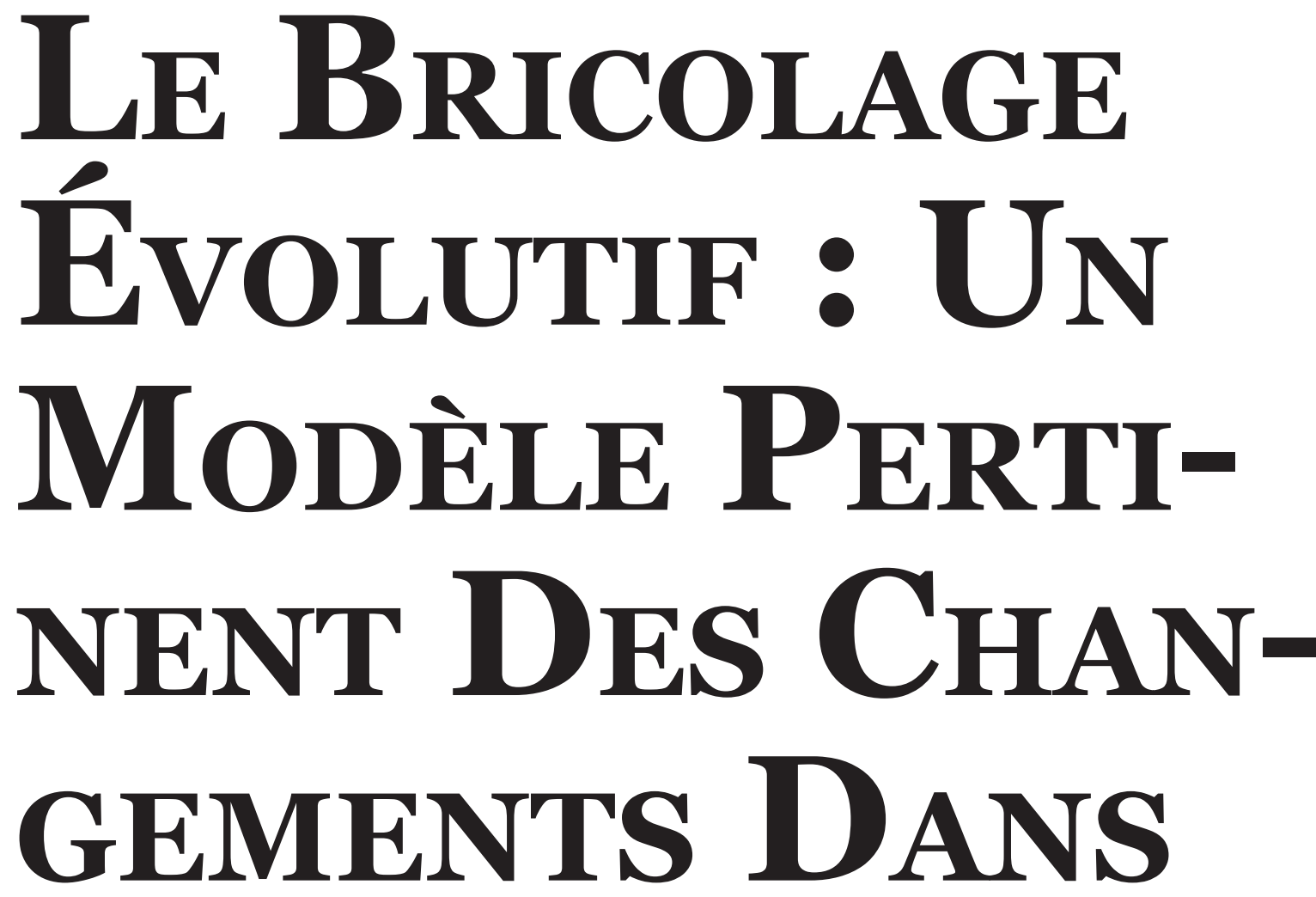

LES SCIENCES

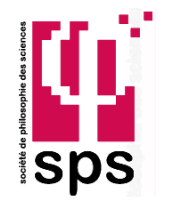

SOCIÉTÉ DE PHILOSOPHIE DES SCIENCES (SPS) 
Ignace Yapi

\section{Le Bricolage Évolutif : Un Modèle Perti- nent Des Changements Dans Les Sciences}

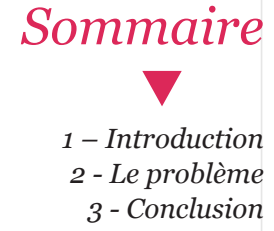

\begin{abstract}
Cet article propose un modèle additionnel pour l'interprétation des changements qui, dans les sciences, ne résultent pas d'une rupture irréversible avec le passé, mais du recyclage opportuniste de vieilles théories, destiné soit à en enrichir les théories émergentes, soit à provoquer le retour contre-révolutionnaire des théories défaites. Il soutient que, quand la science crée ainsi du neuf à partir du vieux, elle procède suivant un modèle proche du «bricolage évolutif », par lequel François Jacob explique l'évolution par le jeu des réaménagements opportunistes qui surviennent dans la structure du noyau cellulaire.
\end{abstract}

This paper proposes a particular pattern for changes in sciences which do not stem from irreversible break with the past, but from recycling old theories, with a view to the growth of new theories or the counter-revolutionary resurgence of defeated ones. It maintains that when science progresses by creating the new from the old, it processes following a pattern akin to the «evolutionary tinkering» which François Jacob associates with the evolution in light of the shifting of opportunistic rearrangements that occur in the cell nucleus structure

Mots clés: bricolage évolutif, contre-révolution, recyclage, résurgence, révolutions scientifiques

Key-words: evolutionary tinkering, counter-revolution, recycling, resurgence, scientific revolutions.

\section{1 - Introduction}

Il peut paraître paradoxal d'affecter le modèle graduel des «bricolages» en œuvre dans l'évolution biologique aux changements brusques, voire brutaux, que constituent les révolutions scientifiques. Celles-ci sont en effet traditionnellement définies comme des moments où une théorie majeure, confrontée à une crise profonde, est destituée et remplacée par une autre. Selon cette interprétation, la science progresse au gré de ses ruptures avec le passé.

Or, certains remaniements théoriques importants se produisent suivant un processus qui concilie rupture et continuité, évolution et révolution. La science, à cette occasion, produit du neuf à partir du vieux en puisant dans le fonds hétéroclite de sa mémoire les éléments de sa reconstruction. Par exemple, l'héliocentrisme de Copernic, l'évolutionnisme de Darwin, l'atomisme de Newton, lointaines réplications d'intuitions pythagoriciennes, épicuriennes, démocritéennes, illustrent toute la force révolutionnaire de la résurgence des théories désuètes. En réalité, le progrès résulte certes de la réhabilitation des vieilles théories abandonnées, mais aussi du recyclage des concepts structurants des théories résurgentes, faute de quoi le retour du passé s'avérerait redondant.

Cette allure à la fois récursive et progressive des changements provoqués par la résurgence et le recyclage des théories n'est guère perceptible dans les modèles traditionnels des révolutions scientifiques. Elle requiert donc un modèle propre qui en souligne les indicateurs spécifiques.

Or, le progrès obtenu par la réhabilitation et le recyclage du vieux dispose d'un modèle en biologie, le « bricolage évolutif », par lequel le généticien François Jacob $(1977,1981)$ explique comment l'évolution ne résulte pas de l'émergence d'entités biochimiques nouvelles, mais de recombinaisons variées de matériaux anciens dans la structure des macromolécules du noyau cellulaire. Les recyclages effectués sur les matériaux conceptuels anciens par la science s'apparentent ainsi aux bricolages opportunistes de l'évolution. Comme la nature, la science accomplit ses changements les plus décisifs par une palingenèse fondée sur la déstructuration et la restructuration des matériaux anciens. Et, bien que l'idée anthropomorphique de bricolage soit plus valorisante pour la nature que pour l'esprit, il y a incontestablement une similitude entre la réutilisation par la première de pièces moléculaires abandonnées au cours de l'évolution pour former de nouveaux organismes, et la réutilisation par le second de pièces conceptuelles démodées pour constituer de nouvelles théories. Celle-ci autorise à rapprocher par un même modèle les changements continus et graduels de la nature et ceux par lesquels la science opère les ruptures les plus profondes dans son processus historique et associer à l'image d'une activité rationnelle de l'esprit celle d'opérations aveugles de la nature, parce que tous sont marqués des mêmes indicateurs d'un processus opportuniste.

\section{2 - Le problème}

Le modèle opportuniste qui explique l'évolution par la nonlinéarité que la nature introduit par ses bricolages dans la structure des entités biochimiques convient à l'interprétation aussi bien des changements scientifiques graduels que nourrit l'apport opportuniste de concepts recyclés, que des « contre-révolutions » provoquées par la résurgence des théories défaites.

\subsection{Le théorème}

Le premier modèle des révolutions scientifiques, bien que 


\section{LE BRICOLAGE Evolu- TIF : Un Modèle Perti- NENT DES ChangementS DANS Les SCIENCES}

tourné vers le passé, n'exprime guère l'idée de résurgence opportuniste. Défendu par les encyclopédistes au XVIII siècle, il est inspiré des révolutions astronomiques.

Ce passage d'un article consacré « l'observation » éclaire sur la notion de « révolution scientifique » chez les encyclopédistes : « La médecine paraît être sur le point d'une grande révolution... Plusieurs médecins s'appliquent comme il faut à l'observation ; ils suivent la nature et ne tarderont pas à faire revivre la médecine d'Hippocrate, qui est la véritable médecine d'observation. Ainsi, après bien des travaux, cette science pourra être avancée et portée au point où elle était il y a 2000 ans » (Diderot et D'Alembert 1751-1772, t. 11, p. 312). Pour l'auteur anonyme de l'article, la véritable référence de la récurrence révolutionnaire est moins la médecine hippocratique que la méthode d'observation qu'elle a su appliquer. Si l'Encyclopédie désigne Hippocrate comme « l'étoile polaire de la médecine » (Ibid., t. 10, p. 265), c'est parce qu'il « a été le premier et le meilleur de tous les médecins observateurs. » (Ibid., t. 11, p. 312) et peut, à ce titre, être proposé « pour modèle à quiconque veut suivre une semblable route, c'est-àdire s'adonner à la partie de la médecine la plus sûre, la plus utile et la plus satisfaisante » (Ibid., t. 11, p. 312).

La récurrence révolutionnaire, dans ces conditions, effectue un parcours normatif visant un idéal intemporel. Elle est plus récursive, voire réflexive, que proprement régressive. Le ressourcement périodique vise le renouvellement cathartique du pacte fondateur, de sorte qu'il n'y a, pour toute science, qu'une révolution, celle qui la rapporte à son idéal et que répètent sans fin les différents retours à cette référence unique ${ }^{1 .}$

Cette figure exaltée du passé récurrent diffère radicalement de la figure recyclée du passé résurgent. Conçu comme le dépositaire d'un idéal perdu, le passé est appelé à revivre sous sa forme intégrale, à l'occasion de chaque épisode révolutionnaire. Or, quand se produisent des résurgences théoriques, on n'assiste pas à la revendication nostalgique d'une idée surannée, mais à la récupération opportuniste de ses fragments recyclés.

Le modèle qui a succédé au modèle astronomique conçoit les révolutions scientifiques à l'image des bouleversements sociopolitiques, comme des changements qui opèrent des ruptures irréversibles avec le passé. En effet, s'il est vrai que les révolutions politiques comme la Révolution française de 1789 paraissent portées à la fois par les idées de ressourcement et de dépassement, de phora et de generis (Saint-Sernin 1993, p. 332), dans le modèle des révolutions scientifiques qu'elles inspirent et qui apparaît sous des modulations spécifiques, chez des auteurs comme Koyré, Popper, Bachelard, Kuhn, l'allure conservatrice et récursive a définitivement fait place à une allure résolument subversive et progressive.

Selon Kuhn qui proposa, en 1962, dans La structure des révolutions scientifiques, la première étude systématique des révolutions scientifiques, celles-ci donnent lieu au renouvellement du patrimoine théorique des sciences. Cependant, résultant d'épreuves où s'affrontent des paradigmes fondamentalement incommensurables, elles n'entraînent ni « progrès » ni « dépassement » dans l'approximation de la vérité : « La concurrence entre paradigmes n'est pas le genre de bataille qui puisse se gagner avec des preuves » (Kuhn 2008, p.204) ; ainsi, « nous devrions peut-être abandonner la notion, explicite ou implicite, selon laquelle, les changements de paradigmes amènent les scientifiques, et ceux qui s'instruisent auprès d'eux, de plus en plus près de la vérité » (Ibid., p. 232). Bien que les théories changent à l'occasion des révolutions, il n'y a pas, " dans leur succession, une direction cohérente de développement ontologique ».

Cette interprétation qui introduit une forme d'irrationalisme au cœur du processus scientifique a été critiquée par d'autres épistémologues, dont Lakatos (Lakatos et Musgrave 1970) qui dénonça une doctrine " mystique » des changements dans les sciences. Popper et Bachelard, quant à eux, mettent en avant l'enjeu du progrès. Toutes les formes de la discontinuité scientifique qu'ils décrivent contribuent, selon eux, au progrès scientifique. Popper se singularise cependant par l'introduction d'une nouvelle clé d'interprétation de la « logique de la découverte » : la falsification des conjectures scientifiques.

Il résulte de ces approches une théorie standard dans laquelle les notions de crise, rupture et dépassement jouent le rôle clé dans la structure des révolutions scientifiques. Or, le progrès par rupture et dépassement implique que le processus scientifique est irréversible, que les états antérieurs des sciences sont, au mieux, associés aux anticipations des précurseurs des théories actuelles, au pis, à des archaïsmes erronés qu'il faut vite oublier.

Le modèle progressif ne saurait convenir aux révolutions lors desquelles la science réhabilite son passé, non pour s'enrichir de ses anticipations, mais pour reconnaitre ses erreurs lorsqu'elle condamnait injustement une théorie valide. Dans celles-ci, ce n'est pas tant le retour au passé que le recours au dépassé qui mettent en exergue, non seulement la réversibilité des révolutions, mais la quasi-normativité de la rationalité scientifique capable de se réapproprier ce qu'elle a d'abord renié. Elles montrent ainsi un caractère absent du modèle progressif : si les victoires scientifiques sont toujours provisoires, les défaites le sont probablement aussi, puisque le dépassement, l'abandon ou le bannissement d'une thèse scientifique ne la condamne plus irrémédiablement; elle peut espérer revenir, à tout moment, sous une forme revisitée.

L'histoire des sciences est riche en théories résurgentes et en concepts mutants. Atome, éther, évolution, infini, inertie, espèce, force, humeur/thymie, transmutation (des métaux), sélection naturelle, sont quelques-uns de ces concepts anciens que la science moderne a adoptés en les dépouillant partiellement ou entièrement de leur représentation originelle.

Mais les résurgences théoriques n'induisent pas toujours un processus révolutionnaire. Le recours au passé, même lointain, peut s'inscrire dans un schéma évolutif, qui ressuscite une vieille idée pour l'intégrer, au prix de quelques réformes, à une théorie régnante ou émergente. Par exemple, quand Aristote assume l'idée pythagoricienne de la circularité des orbes planétaires, il ne retourne pas à l'héliocentrisme, mais intègre ce concept résurgent au système géocentrique. De 


\section{LE BRIColage Évolu- TIF : Un Modèle Perti- NENT DES ChANGEMENTS DANS Les SCIENCES}

même, Copernic emprunte au géocentrisme les épicycles d'Appolonios et de Ptolémée et s'en sert, dans un cadre héliocentrique, pour expliquer les variations des positions et des vitesses angulaires des planètes autour du Soleil.

Il y a cependant d'authentiques résurgences contre-révolutionnaires, comme celles réalisées par Copernic ou Darwin, qui réhabilitèrent des théories déchues et les substituèrent aux théories régnantes. Ce sont elles qui portent les enseignements les plus inattendus sur les processus scientifiques et confirment la validité paradoxale du modèle opportuniste pour l'interprétation des changements non graduels en science.

\subsection{La preuve}

Il faut d'emblée remarquer que l'utilisation d'un modèle biologique pour interpréter des phénomènes non biologiques n'est ni originale ni inédite. Des tentatives existent déjà qui, par exemple, proposent une modélisation biologique des faits culturels et humains (Wilson 1975 ; Cavalli-Sforza 2005a, 2005b ; Jouxtel 2005). Cette tendance qui s'ancre à mesure que se confirment et s'amplifient les succès de la biologie, est défendue par certains et décriée par d'autres qui redoutent le réductionnisme du « tout-biologique » et, singulièrement, du « tout-génétique ».

Cependant, ceux qui sont tentés d'affecter un modèle biologique aux processus historiques visent à en souligner la continuité, étant donné que le modèle biologique ne convient généralement qu'à la représentation de phénomènes graduels et évolutifs. Même s'il peut exister, en biologie, des phénomènes qui s'apparentent à des saltations (les « monstres prometteurs » de Richard Goldschmidt, les " équilibres ponctués » de Stephen Jay Gould, les " transitions en individualité » (Szathmáry et Maynard Smith 1995), les « sauts évolutifs » de Suzanne L. Rutherford et Susan Lindquist, etc., ceux-ci s'intègrent, en définitive, à un processus globalement graduel. De sorte qu'aucun biologiste ne les désignerait comme des « révolutions » biologiques ${ }^{2}$.

Le modèle opportuniste de Jacob est, lui aussi, destiné à décrire un processus graduel : l'évolution. Toutefois, sans en renier la continuité bien caractéristique, il montre qu'elle dépend de la recombinaison de pièces provenant de structures différentes, introduisant par-là une discontinuité dans les structures en évolution : "Gènes et protéines ne sont plus des objets uniques, des idiosyncrasies propres à une espèce... [Ils] sont pour la plupart des sortes de mosaïques formées par l'assemblage de quelques éléments, de quelques motifs portant chacun un site de reconnaissance... C'est la combinatoire de ces motifs qui donnent aux protéines leur infinie variété. C'est la combinaison de quelques motifs particuliers qui donne à une protéine ses propriétés spécifiques » (Jacob 2000, pp. 116-117).

D'après le modèle du bricolage évolutif, introduit par Jacob en 1977 dans son article «Evolution and Tinkering » puis développé en 1981, dans Le jeu des possibles, l'évolution n'opère pas à la manière d'un ingénieur qui travaillerait en fonction d'un plan prédéfini et utiliserait des matériaux adap- tés à son projet, mais à la manière d'un bricoleur qui, sans but, combine divers objets que les circonstances placent à sa portée. L'analyse de la structure des protéines et des gènes montrait en effet d'étranges phénomènes témoignant de la non-linéarité et de la discontinuité dans la structuration des macromolécules biochimiques : "Une structure donnée se retrouvait dans un même organisme avec une fonction différente ", ou « la même structure, ou une structure très voisine, se retrouvait chez des animaux différents », ou enfin « il y avait des morceaux de différentes protéines qui se ressemblaient » (Jacob 1999, p. 18). La diversité biologique naît du bricolage indéfini des mêmes matériaux biochimiques disponibles depuis les origines. Par exemple, l'apparition de nouveaux gènes résulte du jeu varié de réassortiments de séquences nucléides (Jacob 1981, p. 79).

L'évolution suit donc d'un bricolage qu'on observe aussi bien dans les réaménagements moléculaires qu'à l'échelle naturaliste. Darwin, cité par Jacob (Jacob 1999, p. 20), évoquait dans De La fertilisation des Orchidées par les insectes et des bons résultats du croisement (Darwin 2013), l'idée que " dans la nature entière, presque chaque partie de chacun des êtres vivants a probablement servi, dans un état légèrement modifié, à divers usages et a fonctionné dans la machinerie de nombreuses formes vivantes spécifiques anciennes et distinctes ». S'appuyant sur l'existence de nombreux cas d'organes dits « homologues », Jacob confirme la validité de l'hypothèse darwinienne : "Souvent sans dessein à long terme, le bricoleur prend un objet dans son stock et lui donne une fonction inattendue. D'une vieille roue de voiture il fait un ventilateur; d'une table cassée, un parasol. Ce genre d'opération ne diffère guère de ce qu'accomplit l'évolution quand elle produit une aile à partir d'une patte, ou un morceau d'oreille avec un fragment de mâchoire » (Jacob 1981, p. 71). À tous ses échelons, la nature crée du nouveau " en combinant sans fin les mêmes morceaux et les mêmes fragments ».

Résurgence et recyclage, discontinuité structurelle et continuité ontologique caractérisent l'évolution. Pareillement, en science, certains changements sont provoqués par le réinvestissement opportuniste et le recyclage subséquent de théories périmées. La pertinence d'un tel rapprochement est toutefois soumise à une différence importante : si, dans les processus biologiques, les réarrangements structurels concourent au mouvement graduel de l'évolution, dans les contre-révolutions théoriques, les réaménagements conceptuels sont les phases évolutives d'un processus discontinu. À cela près, les indicateurs opportunistes majeurs paraissent présents dans la structure des contre-révolutions scientifiques comme celle que réalisa Copernic lorsque le géocentrisme, après plus de vingt siècles de règne, fut confronté à des impasses et que, pour la remplacer, il n'introduisit point une théorie inédite, mais une ancienne : l'héliocentrisme des pythagoriciens.

Le changement opéré par Copernic repose-t-il sur des indicateurs spécifiques du modèle opportuniste? D'abord, à l'image de la nature, l'astronomie disposait d'un fonds théorique primitif, sur lequel s'effectuèrent, au fil de l'histoire, diverses recombinaisons ou bricolages. Ce fonds était constitué des idées spéculatives de géocentrisme, héliocentrisme, pyrocentrisme, hestiocentrisme, héliostatisme, géostatisme, 


\section{LE BRICOLAge Évolu- TIF : Un Modèle Perti- NENT DES ChangEMENTS DANS Les SCIENCES}

géodynamisme, héliodynamisme, qui formaient sa base de données théorique, disponible au moins à partir $\mathrm{du} \mathrm{V}^{\mathrm{e}}$ siècle avant Jésus-Christ. Les premiers « bricolages » associèrent des doctrines qui, au départ, étaient étrangères les unes aux autres. Le pyrocentrisme de Philolaos fut rapproché de l'héliocentrisme. Les autres doctrines furent combinées aux deux pôles d'une alternative définitivement scellée : d'une part, au géocentrisme furent associés le géostatisme et l'héliodynamisme et, d'autre part, à l'héliocentrisme furent combinés le pyrocentrisme, l'héliostatisme et le géodynamisme. Dans ce tableau, on note cependant que Hicétas développa une théorie bien singulière, qui associa la centralité et la mobilité de la Terre. Ce dualisme fut tellement enraciné que la contestation ou l'assomption des théories périphériques paraissait déterminer celle du noyau dur. Par exemple, Philolaos et Hicetas furent comptés au nombre des défenseurs de l'héliocentrisme, bien que leurs doctrines fussent respectivement seulement pyrocentrique et géodynamique; il avait suffi qu'elles s'opposent à une idée traditionnellement associée au géocentrisme : l'immobilité de la Terre.

Le fonds théorique de l'astronomie était ainsi analogue, dans sa fonction dynamique, au fonds biochimique de l'évolution. Il permettait aussi, en principe, toutes formes de recompositions élémentaires, même si, comme dans l'évolution, certaines donnèrent lieu à des théories viables et d'autres non. Des recompositions hybrides des systèmes du monde furent tentées, sans succès, par Héraclide du Pont qui imagina un système géo-héliocentrisme, ancêtre de celui de Tycho Brahe ou par Hicétas et son disciple Ecphantos, qui crurent pouvoir concilier la centralité de la Terre avec sa rotation diurne. La «sélection naturelle», qui régit aussi le règne des théories, emporta ces monstruosités conceptuelles, handicapées par leur marginalité vis-à-vis des polarités convenues.

La réduction de la diversité théorique primitive de l'astronomie en dualité antagonique limita le processus historique de cette science au triomphe alterné des deux doctrines rivales. Le géocentrisme, probablement plus ancien parce que fondé sur l'observation spontanée des planètes, fut théorisé au VII ${ }^{\mathrm{e}}$ siècle av. J.-C. par Anaximandre et Anaximène. Il fut ensuite soutenu par des philosophes influents tels que Platon et Aristote. Ce dernier le structura par des arguments spéculatifs restés longtemps imparables : la Terre, comme lieu des corps les plus lourds est nécessairement au centre du monde ; elle est immobile parce que, si elle était dotée d'un mouvement orbital ou rotatoire, tous les corps disposés à sa surface s'envoleraient en sens inverse de son mouvement, etc. À cette doctrine s'opposa, à partir du $\mathrm{V}^{\mathrm{e}}$ siècle, l'héliocentrisme, fruit du génie des mathématiciens pythagoriciens ${ }^{3}$. Mais, progressivement, le géocentrisme s'imposa. L'autorité et la force des arguments d'Aristote, la structuration géométrique dont le dota Ptolémée, le parti pris de l'Église en sa faveur, eurent finalement raison des ingénieuses intuitions pythagoriciennes auxquelles Aristarque de Samos donna pourtant, au III ${ }^{\mathrm{e}}$ siècle avant Jésus-Christ, l'image d'une doctrine complète et rigoureuse, plaçant enfin le Soleil au centre du monde.

Copernic réalisa donc, au XVI ${ }^{\mathrm{e}}$ siècle, par une authentique « contre-révolution » scientifique, la résurgence d'une vieille théorie défaite, l'héliocentrisme. Cette réhabilitation fut l'aboutissement d'une démarche heuristique singulièrement opportuniste :

(1) Il constate que, pour diverses raisons, le système géocentrique n'est pas satisfaisant.

(2) Il cherche dans le corpus disponible des cosmologies passées, une théorie pouvant servir d'alternative au géocentrisme.

(3) II la trouve dans l'idée défendue jadis par Philolaos, Hicetas, Héraclide et Ecphantos défendant le mouvement de la Terre (et non, à proprement parler, la centralité et l'immobilité du Soleil).

(4) Il propose alors le mouvement de la Terre à titre de libre conjecture.

(5) La preuve de l'hypothèse héliocentrique est donnée plus tard «par de longues et nombreuses observations » (Copernic 1970, pp. 42-46).

Le recyclage de l'héliocentrisme ancien survint dans la foulée de sa résurgence opportune si bien que le système conçu par Copernic fut finalement éloigné de celui élaboré par ses inspirateurs. Par exemple, il n'y a plus trace ni du Feu Central (Hestia), ni de l'Anti-Terre (Antichthon). Il n'est pas non plus le système hybride d'Héraclide, et Aristarque, dont la théorie est la plus proche de la sienne, n'est même pas mentionné dans la version définitive du De revolutionibus. En revanche, il conserve quelques « fragments » du géocentrisme aristo-ptoléméen qu'il récuse pourtant : épicycles, orbes solides et circulaires, monde clos et fini, etc. ; des morceaux d'un système vaincu sont ainsi recombinés dans le système résurgent. Ces archaïsmes ne seront emportés que plus tard, quand les observations de Brahe prouvèrent l'inexistence des orbes solides, quand les calculs de Kepler montrèrent la forme elliptique des circuits planétaires, quand les démonstrations de Bruno firent exploser les limites de l'univers copernicien.

On peut cependant voir dans le fait que, contrairement à l'évolution qui dispose d'un large patrimoine de matériaux biochimiques primaires à recombiner, le fonds théorique de l'astronomie est limité à l'héliocentrisme et au géocentrisme, une difficulté qui rendrait inopérante l'interprétation opportuniste de la révolution copernicienne. Mais, à vrai dire, le géocentrisme et l'héliocentrisme étaient des méga-théories constituées de diverses sous-théories, elles-mêmes formées d'une multitude de concepts structurants. Dès lors, les bricolages qu'autorise le fonds théorique de l'astronomie sont moins limités qu'il ne paraît. Aujourd'hui se dessine clairement la perspective d'une révolution post-copernicienne. Qui ne sera pas un retour au géocentrisme. L'astronomie contemporaine paraît avoir surmonté l'antique dualisme réducteur. La découverte récente d'exoplanètes et de systèmes solaires autres que le nôtre n'annonce-t-elle pas une cosmologie polycentrique, fondamentalement anticopernicienne, sans retourner au géocentrisme ? L'histoire de l'astronomie ne paraît plus condamnée à la répétition indéfinie des résurgences contre-révolutionnaires, mais préserve ses capacités à faire ressurgir dans des théories nouvelles des fragments recyclés de vieilles intuitions.

On peut multiplier les exemples qui prouvent la fécondité heuristique du modèle opportuniste des changements scientifiques. Par exemple, la révolution darwinienne réhabilita, elle aussi, une doctrine désuète, même si les lignes d'une 
véritable contre-révolution y sont moins nettes que dans la révolution héliocentrique. D’abord, contrairement à Copernic, qui reconnut l'antériorité des pythagoriciens, Darwin ne se réclama d'aucun ancêtre lointain dans l'évolutionnisme. Ce sont uniquement les faits collectés à bord du Beagle qui paraissent avoir inspiré ses convictions évolutionnistes. Cependant, sa théorie, qui ignore l'antériorité épicurienne, n'en possède pas moins les traits d'une lointaine réplication. L'évolution des espèces, l'inexistence de dessein intelligent, la lutte pour la survie, la sélection naturelle, etc. trouvent en effet leurs premières traces dans l'œuvre des épicuriens, et singulièrement chez Lucrèce. Son De rerum natura rapporte que les êtres vivants sont nés de la terre par générations spontanées, à partir d'un assemblage aléatoire d'atomes. Certains étaient " des monstres, qui naissaient avec des traits et des membres étranges : des êtres privés de pieds ou dépourvus de mains, ou encore muets et sans bouche, ou qui se trouvaient être aveugles et sans regard, ou dont les membres captifs demeuraient entièrement soudés au corps et qui ne pouvaient rien faire, ni se mouvoir ni éviter le danger, ni pourvoir à leurs besoins » (Lucrèce 1964, pp. 178-79). La sélection naturelle épargna les plus aptes à survivre, de sorte que «nombreuses furent les espèces qui durent disparaitre et qui ne purent en se reproduisant se créer une descendance ", d'autant qu' " elles offraient aux autres une proie et un butin sans défense ». Seules survécurent quelques espèces aidées par « la ruse, la force ou la vitesse qui, dès l'origine, assurèrent leur protection » (Ibid., p. 178).

Darwin développe la même conception matérialiste de la vie, en rapportant son origine et sa transmission à l'action de forces erratiques, excluant toute intervention divine. Chez lui comme chez Lucrèce, l'évolution n'obéit à aucun principe téléonomique.

La résurgence de l'évolutionnisme grâce à Darwin (précédé de Lamarck, Erasmus Darwin, Wallace) apparaît comme une contre-révolution qui intervient après le long règne du fixisme, qui, sous différentes formes (créationnisme, préformationnisme, spontanéisme), l'avait longtemps relégué. En effet, à l'instar de l'astronomie, la biologie avait donné naissance à deux doctrines rivales : le créationnisme, vraisemblablement plus ancien, en raison de sa résonance religieuse, défendu de nombreux philosophes dont Platon et Aristote, et l'évolutionnisme, soutenu par les épicuriens. Ainsi fut mis en place, en biologie, un fonds théorique primitif constitué des variantes du fixisme et de l'évolutionnisme. Pour des raisons similaires à celles qui prévalurent en astronomie (autorité d'Aristote et soutien de l'Église), le fixisme domina la pensée biologique jusqu'au XIXe siècle et fut assumé par des naturalistes influents comme Linné et Cuvier.

Ce sont les difficultés à trouver, dans un cadre fixiste, l'explication cohérente de phénomènes tels que « les affinités mutuelles des êtres organisés, leurs rapports embryologiques, leur distribution géographique, leur succession géologique, et d'autres faits encore » qui amenèrent Darwin à conclure que " chaque espèce n'a pas été créée indépendamment, mais est descendue, comme les variétés, d'autres espèces » (Darwin 2013, p. 41). La théorie darwinienne porte sur un concept recyclé de l'évolution, épuré par exemple, des allégations cosmogoniques du De rerum natura de Lucrèce. Elle ne contient aucune allusion à la génération spontanée des premiers vivants ni à l'assemblage primitif des atomes.
La clôture du répertoire théorique sur lequel la biologie effectue ses « bricolages » semble limiter son développement historique à l'alternance renouvelée de deux théories rivales. Mais, sous cette apparente clôture, le fonds théorique biologique est constitué de nombreux concepts structurants dont le recyclage est susceptible d'ouvrir à des développements théoriques inédits, voire à libérer du clivage entre fixisme et évolutionnisme. Sauf à considérer que Darwin a clos l'histoire de la biologie, il faut admettre qu'une révolution anti darwinienne est possible. Sera-t-elle condamnée à ressusciter le créationnisme ancien ? Ou, guidée par des expériences nouvelles, s'orientera-t-elle vers des recompositions conceptuelles inédites ? Il faut faire confiance au génie des biologistes, et regarder toutes les velléités anti-darwiniennes sans aucun parti pris qui nous enfermerait à considérer que Darwin et les darwiniens ont clos l'histoire des théories biologiques.

Les révolutions darwinienne et copernicienne montrent que l'histoire des idées scientifiques est certes progressive, mais qu'elle ne décrit pas toujours un mouvement continuellement orienté vers le futur. Elle se présente aussi parfois sous les traits d'un mouvement composé dans lequel la science revisite son passé comme un appui dont elle se sert opportunément pour se reconstruire et se propulser vers son avenir.

\section{3-Conclusion}

L'intérêt épistémologique du modèle opportuniste des changements dans les sciences est indéniable. Sa validité indique que le modèle standard (Koyré, Kuhn, Bachelard, Popper...), construit sur le triptyque «rupture, dépassement, progrès», ne décrit pas, dans toute leur spécificité, les révolutions provoquées par la résurgence de théories désuètes. Elle indique ainsi que, vraisemblablement, il y a une pluralité de logiques que l'esprit met en œuvre lorsqu'il veut se remettre en cause et amorcer, à nouveaux frais, des changements majeurs dans les sciences. En montrant que des révolutions ne détruisent pas irrémédiablement les théories dépassées, mais les relèguent temporairement, en révélant la force évolutive du bricolage conceptuel, elle donne des révolutions une image qui bouleverse l'allure traditionnelle des processus qui impulsent les progrès dans les sciences.

Le modèle du bricolage évolutif confirme toute l'ambiguïté de la nouveauté scientifique. Celle-ci ne se rapporte pas uniquement à ce qui survient pour la première fois, mais surtout à ce qui suscite un nouveau regard chez les scientifiques, à l'exemple du balancement anodin du lustre de la cathédrale de Pise, phénomène maintes fois aperçu, qui devint un phénomène nouveau pour la science quand Galilée y arrêta son regard et y découvrit l'expression de l'isochronisme du mouvement pendulaire. L'idée de nouveauté pertinente pour interpréter les processus scientifiques qualifie les relations évolutives de l'homme à la nature et à ses propres théories. Il n'est donc pas nécessaire qu'une théorie soit inédite pour constituer une solution adéquate aux impasses scientifiques, comme en témoigne la féconde épopée des théories résurgentes et des concepts mutants.

La pertinence heuristique du bricolage conceptuel n'occulte pas cependant sa différence fondamentale avec le bricolage 


\section{LE BRICOLAGE Évolu- TIF : Un Modêle Perti- NENT DES Changements Dans Les Sciences}

biologique : désignant des opérations volontaristes, motivées par une visée claire, il se distingue, sous ce trait, des manipulations aléatoires de la sélection naturelle. Il n'introduit pas, comme on pourrait l'en accuser, la moindre irrationalité dans les processus scientifiques, ni même un opportunisme semblable à celui qui, par exemple, caractérise la sérendipité.

\section{RÉFÉRENCES}

CAVALLI-SFORZA, Lugi Luca. 2005a. Évolution biologique, évolution culturelle. Paris : Odile Jacob.

CAVALLI-SFORZA, Lugi Luca. 2005b. Des gènes aux idées : l'évolution des cultures. Sciences Humaines, 1, 66-71. COPERNIC, Nicolas. 1970. Des révolutions des orbes célestes. Traduit par A. Koyré. Paris : Blanchard.

DARWIN, Charles. 2013. L'origine des espèces. Traduit par T. Hoquet. Paris : Éditions du Seuil.

DIDEROT, Denis et D'ALEMBERT, Jean Le Rond. 1751-1772. Encyclopédie ou Dictionnaire raisonné des sciences, des arts et des métiers. t.10, t.11, Paris : Briasson-David-Le BretonDurand.

JACOB, François. 1977. Evolution and Tinkering. Science, 196, 1161-1166. Lien

JACOB, François. 1981. Le jeu des possibles. Essai sur la diversité du vivant. Paris : Fayard.

JACOB, François. 1999. Éloge du darwinisme. Magazine littéraire, 374.

JACOB, François. 2000. La souris, la mouche et l'homme. Paris : Odile Jacob.

JOUXTEL, Pascal. 2005. Comment les systèmes pondent.

\section{HISTORIQUE}

Article initialement soumis le 31 octobre 2016.

Article révisé soumis le 18 octobre 2017.

Article accepté le 18 octobre 2017.

\section{SITE WEB DE LA REVUE}

sites.uclouvain.be/latosensu/index.php/latosensu/index

ISSN 2295-8029

DOI HTTP://DX.DOI.ORG/10.20416/LSRSPS.V5I1.4

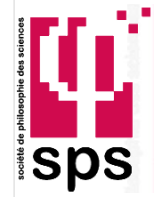

SOCIÉTÉ DE PHILOSOPHIE DES SCIENCES (SPS)

École normale supérieure

45, rue d'Ulm

75005 Paris

www.sps-philoscience.org
Introduction à la mimétique. Paris : Le Pommier.

KUHN, Thomas. 2008. La structure des révolutions scientifiques. Traduit par Laure Meyer. Paris : Flammarion.

LAKATOS, Imre \& MUSGRAVE, Alan (éd.). 1970, Mysticism and the Growth of Knowledge. Cambridge : Cambridge University Press.

LUCRÈCE. 1964. De la nature. Traduit par H. Clouard. Paris : Flammarion.

SAINT-SERNIN, Bertrand. 1993. Crise et révolution selon A.A. Cournot. Revue de métaphysique et de morale, 3. Paris : PUF. 331-346.

SHAPIN, Steven. 1998. La révolution scientifique. Traduit par Claire Larsonneur. Paris : Flammarion.

SZATHMÁRY, Eörs \& MAYNARD SMITH, John. 1995. The major Transitions in Evolution. Oxford : Oxford University Press.

WILSON, Edward O. 1975. Sociobiology: The New Synthesis. Cambridge (Mass.) : Belknap Press of Harvard University Press.

\section{CONTACT ET COORDONNÉES :}

Ignace YAPI

o6 BP 6295 Abidjan 06

Côte d'Ivoire

yapiaci@yahoo.fr

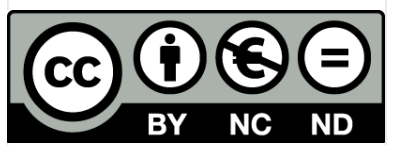

